\title{
A case report of severe veno-occlusive disease following autologous stem cell transplantation successfully treated with Defibrotide
}

\author{
Golamreza Bahoush, Maryam Vafapour \\ Ali-Asghar Children Hospital, Faculty of Medicine, Iran University of Medical Sciences, \\ Tehran, Iran.
}

This article is distributed under the terms of the Creative Commons Attribution Noncommercial License (CC BY-NC 4.0) which permits any noncommercial use, distribution, and reproduction in any medium, provided the original author(s) and source are credited.

\begin{abstract}
Veno-occlusive disease (VOD) is one of the complications of hematopoietic stem cell transplantation that can also be caused by high-dose chemotherapy. This complication can lead to high mortality following bone marrow transplantation. It is more common after allogeneic stem cell transplantation, and is rare after autologous stem cell transplantation. While mild cases of VOD may reduce over a period of a few weeks, very severe cases can cause multi-organ damage, which has a high mortality. is therefore required with early diagnosis and treatment of this complication. In this paper, we present a sever VOD case after autologous stem cell transplantation, that was treated successfully with Defibrotide. The patient was a 14-month-old girl who has neuroblastoma with bone metastasis. VOD should be considered in the differential diagnosis of haematopoietic stem cell transplantation recipients who present with unexplained liver injuries, ascites and/or multi organ failure. Recipients of haematopoeitic stem cell transplantation who present with unexplained liver injuries, ascites and/or multi organ failure should have VOD considered in their differential diagnosis. If there is severe VOD diagnosed, then Defibrotide could be an option for treatment.
\end{abstract}

Key Words: neuroblastoma, hematopoietic stem cell transplantation, veno-occlusive disease, Defibrotide.

Eur J Transl Myol 2020;30 (3): 9161. doi10.4081/ejtm.0.9161

Following hematopoietic stem cell transplantation, patients may develop some complications, one of which is the hepatic sinusoidal obstruction syndrome (SOS), previously termed hepatic Veno-Occlusive Disease (VOD). It usually occurs during the first 21 days of the transplant, however it can also occur after chemotherapy alone. ${ }^{1}$ The incidence of the disease in children after hematopoietic stem cell transplantation (HSCT) has been reported to $22-30 \%$, but in high-risk groups it may increase up to $60 \%{ }^{2}$ There are various risk factors for this complication, such as transplant factors (e.g., the toxicity of chemotherapy, allogeneic transplant), patient profile (e.g., age, female gender, underlying disease, genetic aptitude), and hepatic conditions (e.g., previous liver disease, immature liver function in infants, iron overload, liver fibrosis, hepatitis). ${ }^{3,4}$ The pathogenesis of this disease involves a number of inflammatory and thrombotic factors that cause damage to the sinusoidal endothelial cells of the liver. ${ }^{5}$ Following narrowing of the the lumen of veins, post-sinusoidal portal hypertension occurs and can progress to the clinical symptoms of VOD (See Figure 1 for details of the pathogenesis). ${ }^{5}$ Diagnosis of VOD is based on clinical evidence and examination. Recently, the European Society for Blood and Marrow transplantation (EBMT) has provided a diagnostic guideline and severity grading criteria for the disease in adults and children. ${ }^{2}$

If two or more of the following criteria are present the diagnosis is at least higly suspected:

- Unexplained consumptive and transfusion refractory thrombocytopenia.

- Unexplained weight gain in three consecutive days, despite the use of diuretics or a weight gain $45 \%$ above baseline value.

- Hepatomegaly above baseline value.

- Ascites above baseline value.

- Bilirubin rise from baseline on three consecutive days or bilirubin $\geq 2 \mathrm{mg} / \mathrm{dL}$ within $72 \mathrm{hrs}$. 


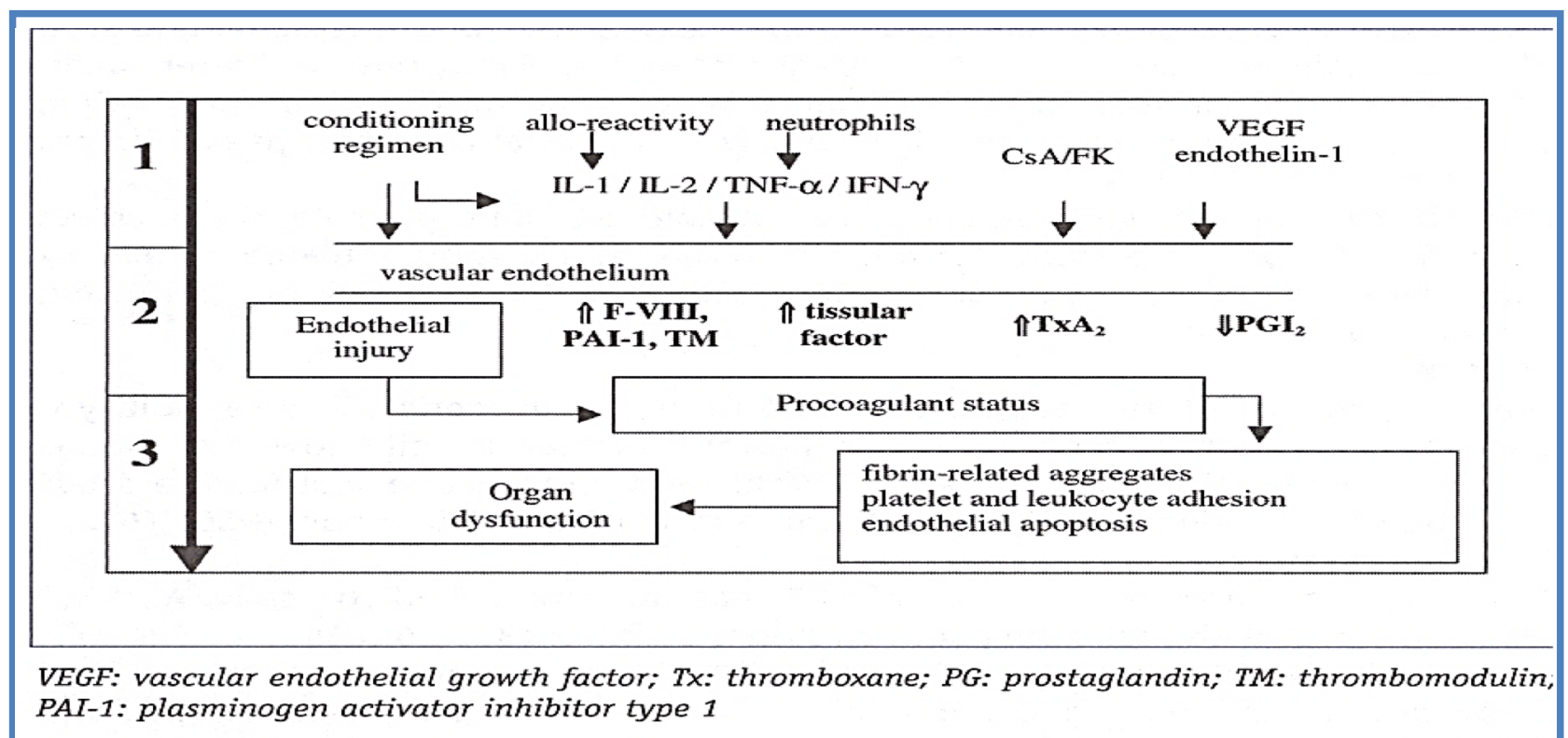

Fig 1. Pathogenesis of VOD

These new diagnostic guidelines provide the criteria for early diagnosis and treatment of the disease. When clinical criteria are not sufficient to a diagnose a VOD, but the suspicion is high, a liver biopsy may be used to confirm or not the diagnosis. ${ }^{6}$ In patients over one-month of age Defibrotide has been approved to treat this condition in the United States and the European Union. ${ }^{1}$ Defibrotide is a polydeoxyribonucleotide that has antiinflammatory effects that protects endothelial cells, thus helping to restore thrombo-fibrinolytic balance. ${ }^{7}$ Therefore, the aim of this report was to the present in a



Fig 2. Ascitic distention of patient's abdomen 14-month-old girl a case of sever VOD after autologous stem cell transplantation that was successfully treated with Defibrotide.

\section{Case Presentation}

A 14-month-old girl, who had neuroblastoma with bone metastasis, had been treated with neoadjuvant chemotherapy with the Vincristine, Prednisolone, Etoposide and Chlorambucil (OPEC) protocol for two courses. She then underwent complete excision of an abdominal mass and left kidney surgery. Then chemotherapy followed (Topotecan/Endoxan) for 5 cycles. Because iodine-131-meta-iodobenzylguanidine (MIBG) was normal, she was candidate for haematopoietic stem cell transplantation (HSCT) at the age of 24 months. In 2017, she underwent autologous stem cell transplantation. Having good conditions, she was discharged on day 13 . On day 28 , the patient returned to hospital with symptoms of abdominal distention (Figure 2) and pain, malaise, fever, weight gain, vomiting and low urine output. During the examination, she had abdominal ascites and tender hepatomegaly. She was admitted to the hospital with a possible diagnosis of VOD. Laboratory studies indicated that she had high serum aminotransferases, bilirubin and thrombocytopenia (Figure 3). Abdominal sonography showed marked hepatomegaly and mild to moderate ascites. Serologic analyses for hepatitis and cytomegalovirus (CMV) yielded negative results.

During hospitalization on day 29, the patient developed respiratory distress, low oxygen saturation, tachypnea, 


\section{Severe Veno-Occlusive Disease}

Eur J Transl Myol 2020; 30 (3): 9161. doi: 10.4081/ejtm.2020.9161

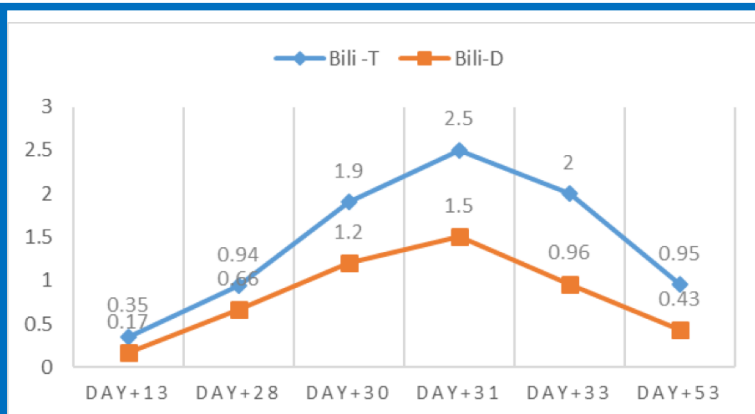

Bili T, Bilirubin Total; Bili D, Bilirubin Direct.

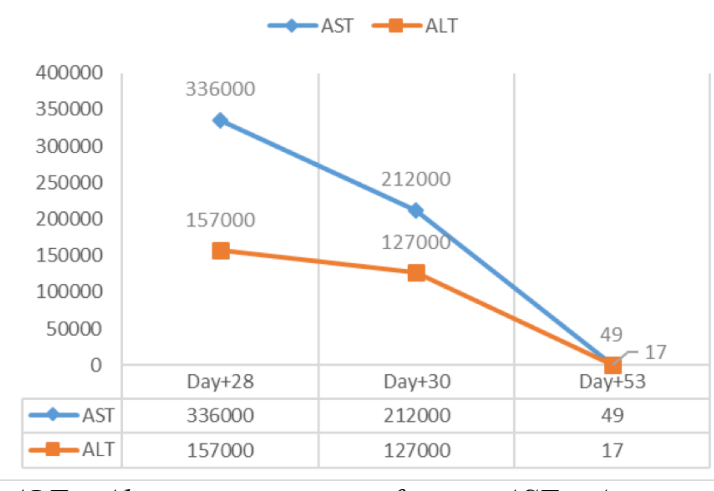

ALT, Alanine aminotransferase AST, Aspartate aminotransferase

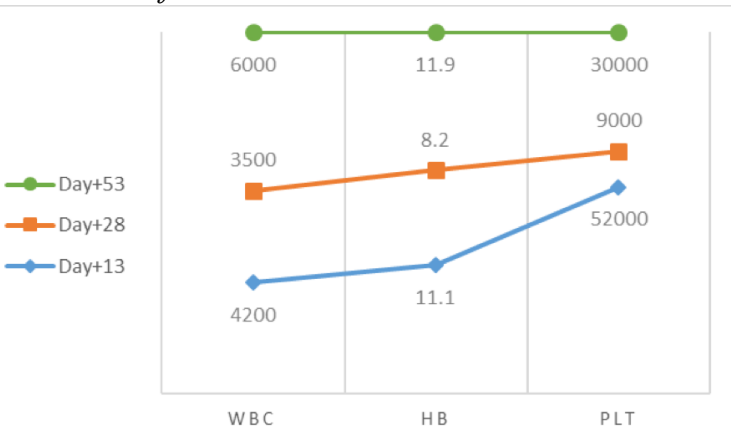

WBC, white blood cell; HB, hemoglobin; PLT, Platelets

Fig 3. Laboratory data

and tachycardia. She was transferred to the intensive care unit. She was treated with antibiotics, heparin, $N$ acetylcysteine, low dose dopamine, albumin and a diuretic. According to serial laboratory tests, the patient's bilirubin was rising. On day 31 , because of the severe VOD, treatment with Defibrotide $(25 \mathrm{mg} / \mathrm{kg} /$ day $)$ was commenced. Defibrotide treatment for 21 days was administered. She responded well to treatment and was discharged in a good general condition.

\section{Discussion}

SOS/VOD is one of the most dangerous complications after HSCT. ${ }^{3,5,6}$ Recognition of this disease and its predisposing factors is important for early diagnosis and treatment. $^{3,5,6}$
Therefore, having diagnostic criteria would be helpful in patient management. The EBMT group has provided diagnostic criteria and divides the risk factors into several groups, including: transplant, hepatic, patient and disease-related. These diagnostic criteria were used in the diagnosis of our patient. She had five criteria, which were: hepatomegaly confirmed by imaging, ascites, weight gain, increase bilirubin and thrombocytopenia. Multi-organ dysfunction (MOD) in VOD is most considered when associated with renal and/or pulmonary dysfunction. Its survival rate is $20-30 \%$ in HSCT patients receiving supportive care alone. ${ }^{1,8}$ Reports of Defibrotide for both the prophylaxis and treatment of VOD suggest that this drug may have a role in reducing the incidence and mortality of this complication. A systematic review study was conducted in 2017 by Richardson and colleagues, on the treatment of VOD with Defibrotide. ${ }^{1}$ They analyzed 17 studies with 2598 patients and investigated survival of patients on day 100 after transplantation. The results of the study were as follows. The pooled survival rate at day 100 was $54 \%$. Use of Defibrotide increased survival rate at day 100 to $56 \%$ (most studies have used the dose of Defibrotide of $25 \mathrm{mg} / \mathrm{kg} /$ day). Survival in patients with VOD without MOD was greater $(69 \%)$ than in patients with combined VOD and MOD (42\%). The definitions used for multiorgan disorder in these studies were as follows: renal dysfunction was considered when creatinine $\geq 3 \times$ level at time of transplant, or creatinine clearance/GFR $\leq 40 \%$ of baseline, or dialysis dependence; pulmonary dysfunction was defined by saturation $\mathrm{O}_{2} \leq 90 \%$ on room air or by the need for supplemental oxygen/ventilator dependence. ${ }^{1}$ According to this systematic review, the patients with VOD and multi-organ dysfunction had higher mortality, and those who used Defibrotide in their treatment had higher survival. ${ }^{1}$ Early onset of treatment also contributed to increased survival. The effect seen in the dose of $25 \mathrm{mg} / \mathrm{kg} /$ day of Defibrotide was not different from the higher doses. Due to the anti-thrombotic, antiischemic and thrombolytic properties of Defibrotide and the pathophysiology of VOD, Defibrotide is thought to be effective in the prophylaxis of VOD. Studies have been conducted to evaluate the side effects of Defibrotide. The overall result was that Defibrotide is a safe drug and the side effects seen in treated patients were not different from those in the control group. Most of the side effects reported with this drug included hemorrhage and hypotension. Common hemorrhagic complications include pulmonary and gastrointestinal bleeding that was not different from the control group. ${ }^{9}$ In a randomized 


\section{Severe Veno-Occlusive Disease}

Eur J Transl Myol 2020; 30 (3): 9161. doi: 10.4081/ejtm.2020.9161

controlled trial, Defibrotide was used for prophylaxis in 356 high risk pediatric patients and the result of the study was that in the treatment group, incidence of VOD was $12 \%$ versus $20 \%$ in the placebo group (risk difference $7 \cdot 7 \%, 95 \% \mathrm{CI}:-15 \cdot 3$ to $-0 \cdot 1$; $\log$-rank test $\mathrm{p}=0 \cdot 0507) .{ }^{10}$ Work by Strouse and colleagues in 2016 has confirmed previous results. ${ }^{8}$ The study also evaluated the incidence of graft-versus-host disease (GvHD) in VOD patients and showed the protective effect of Defibrotide on the incidence and severity of acute GvHD (aGvHD). The results were that the incidence of grade III-IV aGVHD on day 100 was lower in the groups treated with Defibrotide than in the untreated groups. The day 100 cumulative incidence of aGvHD in the treated group was $23.1 \%$ while it was $37.7 \%$ in the untreated group, with an absolute difference of $-14.6 \%$ (95\% CI: $-33.6 \%$ to $3.9 \%$ ). Corresponding rates for grade III-IV aGVHD were $10.9 \%$ of the treated group and $28.6 \%$ for untreated groups, with an absolute difference of $-17.7 \%$ (95 CI: $-33.1 \%$ to $-2.0 \%$ ). They attributed this result to the down regulation of heparanase or inhibition of $\mathrm{T}$ cell activity with the use of Defibrotide. ${ }^{10}$ The patient in the present Case Report had no common risk factors for VOD; she was not suffering from an underlying liver disease before transplant (normal serum transaminase level, no active viral hepatitis). She had undergone an autologous transplant, where VOD is less common. The combination of clinical features and laboratory studies prompted us to think about VOD and start Defibrotide shortly after the onset of symptoms. In Europe, Defibrotide is the only drug approved for the treatment of VOD. It has been shown to have antithrombotic and anti-inflammatory effects and may promote revascularization inducing endothelial cell proliferation and angiogenesis. ${ }^{11}$

In conclusion, VOD should be considered in the differential diagnosis of HSCT recipients who present with unexplained liver injuries, ascites and/or multi organ failure. This case study has suggested that one way in improving clinical outcomes for patients is using therapeutic treatment with Defibrotide. Specifically, early therapeutic intervention with defibrotide can improve the clinical outcomes of these patients.

\section{List of acronyms}

ALT- alanine aminotransferase

AST - aspartate aminotransferase

Bili D - bilirubin direct

Bili T - bilirubin total

CMV - cytomegalovirus

EBMT - European society for blood and marrow transplantation

GVHD, Graft-Versus-Host Disease
HB - hemoglobin

HSCT - hematopoietic stem cell transplantation

MIBG - Metaiodobenzylguanidine

MOD - multi-organ dysfunction

OPEC - vincristine, prednisolone, etoposide and chlorambucil

PAI1 - plasminogen activator inhibitor type 1

PG - prostaglandin

PLT - platelets

SOS - hepatic sinusoidal obstruction syndrome

TM - thrombomodulin

Tx - thromboxaned

VEGF - vascular endothelial growth factor

VOD - veno-occlusive disease

WBC - white blood cell

\section{Authors contributions}

Gr. Bahoush planned the study and edited final manuscript. M.Vafapour collected data and wrote the preliminary manuscript.

\section{Acknowledgments}

We would like to thank both our patient and her family for letting us share their experience.

\section{Funding None}

\section{Conflict of Interest}

The authors declare they have no financial, personal, or other conflicts of interest.

\section{Ethical Publication Statement}

We confirm that we have read the Journal's position on issues involved in ethical publication and affirm that this report is consistent with those guidelines.

\section{Corresponding Author}

Golamreza Bahoush, Ali-Asghar Children Hospital, Faculty of Medicine, Iran University of Medical Sciences, Tehran, Iran.

ORCID iD: 0000-0003-1463-9369

Email: bahoush.gh@iums.ac.irm

Email and ORCID iD of Coauthor

Laris Maryam Vafapour: Vafapourm@gmail.com ORCID iD: 0000-000223268363

\section{References}

1. Richardson P, Aggarwal S, Topaloglu O, et al. Systematic review of defibrotide studies in the treatment of veno-occlusive disease/sinusoidal obstruction syndrome (VOD/SOS). Bone Marrow Transplant 2019;54;1951-62. doi: 10.1038/s41409019 0474-8

2. Corbacioglu S, Carreras E, Ansari M, et al. Diagnosis and severity criteria for sinusoidal obstruction syndrome/veno-occlusive disease in pediatric patients: a new classification from the European Society for Blood and Marrow Transplantation. Bone Marrow Transplant 


\section{Severe Veno-Occlusive Disease}

Eur J Transl Myol 2020; 30 (3): 9161. doi: 10.4081/ejtm.2020.9161

2018;53:138-45. doi: 10.1038/bmt.2017.161

3. Mohty M, Malard F, Abecassis M, et al. Sinusoidal obstruction syndrome/veno-occlusive disease: current situation and perspectives-a position statement from the European Society for Blood and Marrow Transplantation (EBMT). Bone Marrow Transplant 2015;50:781-9. doi: 10.1038/bmt.2015 .52

4. Carreras E, Diaz-Ricart M. The role of the endothelium in the short-term complications of hematopoietic SCT. Bone Marrow Transplant 2011;46:1495-502. doi: 10.1038/bmt.2011.65

5. Mohty M, Malard F, Abecassis M, et al. Revised diagnosis and severity criteria for sinusoidal obstruction syndrome/veno-occlusive disease in adult patients: a new classification from the European Society for Blood and Marrow Transplantation. Bone Marrow Transplant 2016;51:906-12. doi: 10.1038/bmt.2016.130

6. Castellino A, Guidi S, Dellacasa CM, et al. LateOnset Hepatic Veno-Occlusive Disease after Allografting: Report of Two Cases with Atypical Clinical Features Successfully Treated with Defibrotide. Mediterr J Hematol Infect Dis 2018;10: e2018001. doi: 10.4084/mjhid.2018.001

7. Palomo M, Mir E, Rovira M, et al. What is going on between defibrotide and endothelial cells? Snapshots reveal the hot spots of their romance. Blood 2016;127:1719-27. doi: 10.1182/blood-
2015-10-676114

8. Strouse C, Richardson P, Prentice G, et al. Defibrotide for treatment of severe veno-occlusive disease in pediatrics and adults: an exploratory analysis using data from the Center for International Blood and Marrow Transplant Research. Biol Blood Marrow Transplant 2016;22:1306-12. doi: 10.1016/j.bbmt.2016.04.011

9. Richardson PG, Riches ML, Kernan NA, et al. Phase 3 trial of defibrotide for the treatment of severe veno-occlusive disease and multi-organ failure. Blood 2016;127:1656-65. doi: 10.1182/ blood-2015-10-676924

10. Corbacioglu S, Cesaro S, Faraci M, et al. Defibrotide for prophylaxis of hepatic venoocclusive disease in paediatric haemopoietic stemcell transplantation: an open-label, phase 3, randomized controlled trial. Lancet 2012;379:1301-9. doi: 10.1016/S0140-6736(11) 61938-7

11. Richardson PG, Elias AD, Krishnan A, et al. Treatment of severe veno-occlusive disease with defibrotide: compassionate use results in response without significant toxicity in a high-risk population. Blood 1998; 92:737-44. doi: 92(3):73744

Submission: June 4, 2020

Revision received: June 15, 2020 Accepted for pubblication: June 18, 2020 